\title{
Are coffee silverskin extracts safe for topical use? An in vitro and in vivo approach
}

\author{
F. Rodrigues, C. Pereira, F.B. Pimentel, R.C. Alves, M. Ferreira, B. Sarmento, \\ M. Helena Amaral, M. Beatriz P.P. Oliveira
}

\begin{abstract}

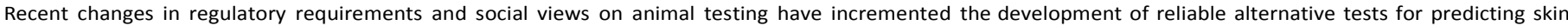

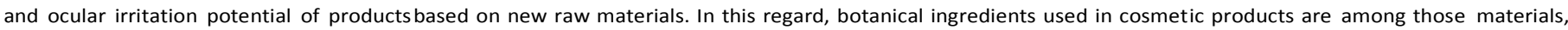

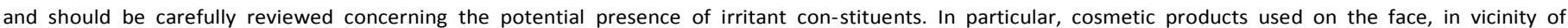

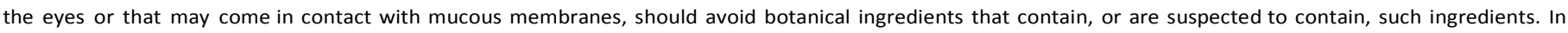

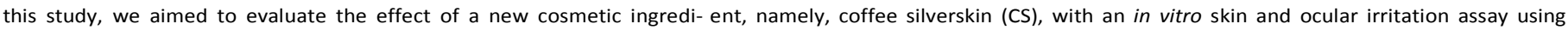

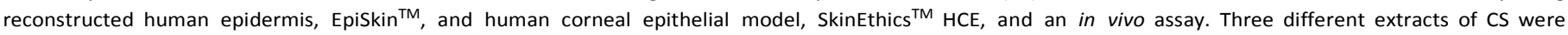

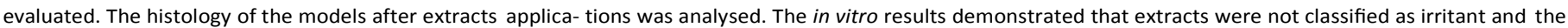

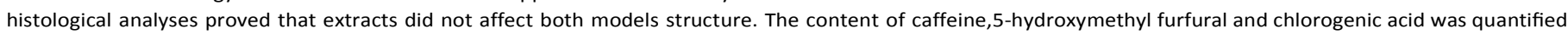

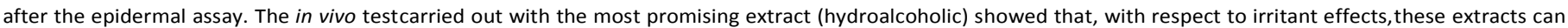
be regarded as safe for topical application.
\end{abstract}

Keywords:

In vitro skin cultures, Interleukin-1a (IL-1a), Skin irritation potential, Coffee silverskin, EpiSkin ${ }^{\mathrm{TM}}$

SkinEthics ${ }^{\mathrm{TM}} \mathrm{HCE}$

\section{Introduction}

In recent years, sustainable approaches have become an essen- tial challenge for different industries. Answering to this issue, the present study was undertaken to investigate the potential use of coffee silverskin (CS) extracts in cosmetic products. Usually, per coffee fruit are found two coffee beans, each one covered by a thin closely skin called silverskin (Saenger et al., 2001). CS is a main by- product of the coffee roasting procedure and has no commercial value, being discarded as a solid waste (Saenger et al., 2001). This may have negative effects on the environment requiring proper management. Recent advances in industrial biotechnology leads to potential opportunities for economic valorization of this by- product (Pandey et al., 2000). Some work has been performed on the properties of $\mathrm{CS}$, in particular its antioxidant behaviour, which reveals a good content (Borrelli et al., 2004; Costa et al., 2014; Narita and Inouye, 2012; Panusa et al., 2013; Pourfarzad et al., 2013; Rodrigues et al., 2015). An example is chlorogenic acid (CGA), which is a highly valuable natural polyphenol com- pound used in medicine and industries (Sato et al., 2011). However, some process steps in coffee production could affect CGA content, such as roasting, decaffeination and/orblending (Mills etal., 2013). Also, the caffeine content should be very high and similar to that of coffee beans (Bresciani et al., 2014; Narita and Inouye, 2012; 
Pourfarzad et al., 2013). These compounds are believed to provide in vivo protection against free radical damage. As coffee beans, CS contains several classes of health compounds such as phenolics, diterpenes, xanthines, and vitamin precursors (Alves et al., 2009; Ludwig et al., 2012). Caffeine is being increasingly used in cos- metics due to its high biological activity and ability to penetrate the skin barrier (Herman and Herman, 2013). A number of claims, as anticellulite properties, are based on the implicit assumption that this bioactive substance is effectively released from the for-mulation into epidermis and probably through epidermis into the dermal and subcutaneous tissues, preventing the excessive accu- mulation of fat in cells, and providing a slimming effect (Bolzinger et al., 2008; Herman and Herman, 2013). This alkaloid stimulates the degradation of fats during lipolysis through inhibition of the phosphodiesterase activity and has potent antioxidant properties (Herman and Herman, 2013). However, studies assessing the skin absorption of caffeine released from extracts are extremely rare in the literature. Another compound that should be taken in con- sideration regarding CS is 5-hydroxymethyl furfural (HMF), which is formed during coffee roasting (del Campo et al., 2010). HMF is cytotoxic, irritating to the eyes, skin and mucous membranes at high concentrations, being very important to quantify its presence in CS extracts (Capuano and Fogliano, 2011).

The assessment of irritation is one of the primary procedures to evaluate and hazard classify a substance, particularly, in cosmet- ics or pharmaceuticals (Cotovio et al., 2010). In line with the 7th amendment deadlines, European Union bans the in vivo skin irri-

tation assessment on ingredients for cosmetic purposes, regarding concerns about the test's reproducibility, plus animal welfare and political pressures (Draize et al., 1944; Spielmann et al., 2007). A number of in vitro tests to assess potential skin or eye irritants have been developed. The reconstructed human epidermis EpiSkin ${ }^{\mathrm{TM}}$ test method was validated by European Union Reference Laboratory for alternatives to animal testing (ECVAM) as replacement test

for the prediction of acute skin irritation. According to Cotovio et al. (2010), the assessment of ocular irritation is also one of the primary procedures to evaluate and hazard classify a new substance. The in vivo Draize irritation rabbit eye test continues to be described in the current OECD test guidelines, but ethical questions are lead- ing to the development of in vitro alternative tests, such as Human Corneal Epithelial Model (SkinEthic ${ }^{\mathrm{TM}} \mathrm{HCE}$ ), which are still under validation by ECVAM.

The aim of this study is to evaluate the in vitro and in vivo irrita- tion potential of three CS extracts. For both EpiSkin ${ }^{\mathrm{TM}}$ and SkinEthic $\mathrm{HCE}^{\mathrm{TM}}$ assays, MTT and IL- $1 a$ were used as endpoints. After the extract contact with the model, the histology of the model was eval- uated. To ensure the possible content of caffeine, CQA and HMF that pass through RhE, an HPLC assay was developed. In vivo skin irri- tation potential observed after single application under occlusion was assessed along with sodium lauryl sulfate (SLS) solution $(2 \%, w / v)$ as irritant model (positive control) and water as non-irritant (negative control).

\section{Materials andmethods}

\subsection{Chemicals}

Coffee silverskin samples were provided by a national coffee roaster (BICAFÉ - Torrefação e Comércio de Café Lda, Portugal). EpiSkin $^{\mathrm{TM}}$ and SkinEthic ${ }^{\mathrm{TM}}$ HCE model were purchased from SkinEthic Laboratories (Lyon, France). 3-[4,5-Dimethylthiazol-2- yl]2,5-diphenyltetrazolium bromide (MTT) and sodium lauryl sulfate (SLS; purity >99\%) were purchased from Sigma-Aldrich Chemical (St Quentin Fallavier, France). ELISA Quantikin kit for IL-1a measurements was from R\&D Systems (Lille, France).
Deionised water was obtained using Mili- $Q$ water purification sys-tem (TGI Pure Water Systems, USA). Ethanol was obtained from Panreac (Barcelona, Spain). HPLC-grade acetonitrile was from Fluka (Madrid, Spain). 5-(Hydroxymethyl)furfural (HMF), caffeine and chlorogenic acid (CGA) standards were from Sigma-Aldrich (Stein- heim, Germany). Metaphosphoric acid, isopropanol, phosphate buffered saline (PBS; $\mathrm{pH}$ 7.4) and paraformaldehyde were from Sigma-Aldrich (St. Louis, Missouri, USA).

\subsection{Preparation of extracts}

CS was milled to particle size of approximately $0.1 \mathrm{~mm}$ using a A11 basic analysis mill (IKA Wearke, Staufen, Germany) and stored in silicone tubes at room temperature $\left(25-28{ }^{\circ} \mathrm{C}\right)$ until extract preparation, as described by different authors (Costa et al., 2014; Rodrigues et al., 2015). Samples (1 g) were submitted to solvent extraction by maceration with $20 \mathrm{~mL}$ of water, ethanol:water $(1: 1, \mathrm{v} / \mathrm{v})$ or ethanol for $30 \mathrm{~min}$ at $40{ }^{\circ} \mathrm{C}$. The three different extracts obtained were filtered through Whatman No. 1 paper filter and the filtrates collected (Costa et al., 2014).

\subsection{In vitro models assays}

In order to determine the irritant potential of CS extracts on skin and eye, in vitro tests were performed. Three different extracts mentioned above were tested.

The EpiSkin model (large model) was obtained from a standardized/arge-scale production certified by the ISO 9001 standard. EpiSkin units were delivered to the laboratory within $24 \mathrm{~h}$. Upon arrival, tissues were transferred to 12 well plates containing $37^{\circ} \mathrm{C}$ pre-warmed maintenance media $(2 \mathrm{~mL} /$ well) and incubatedovernight at $37^{\circ} \mathrm{C}, 5 \% \mathrm{CO}_{2}$ and $95 \%$ relative humidity.

The SkinEthic HCE model is composed of immortalized human corneal epithelial cells cultured in a chemically defined medium and seeded on a synthetic membrane at the air-liquid interface. The tissue is represented by a multilayered epithelium, with five to seven cell layers and a surface area of $0.5 \mathrm{~cm}^{2}$. The assay was performed according to the manufacturer instructions (Alépée et al., 2013; Cotovio et al., 2010). Upon arrival, tissues were transferred to 24 well plates containing $37^{\circ} \mathrm{C}$ pre-warmed maintenance media $\left(1 \mathrm{~mL} /\right.$ well) and incubated $24 \mathrm{~h}$ at $37{ }^{\circ} \mathrm{C}, 5 \% \mathrm{CO}_{2}$ and $95 \%$ relative humidity.

\subsubsection{Skin irritation test}

The in vitro reconstructed human skin tissue (EpiSkin ${ }^{\mathrm{TM}}$ ) method, proposed to replace animal testing for skin corrosivity and skin irritation (Tornier et al., 2010), is based on determining cell via- bility, and cytokine release (IL-1 $a$ ) as an additional endpoint. The reconstructed $0.38 \mathrm{~cm}^{2}$ skin inserts were used and the assay was performed according to the manufacturer instructions and proto- col. Negative (PBS-treated) and positive controls $(5 \%(\mathrm{w} / \mathrm{v})$ of SLS) were used. After exposing to the extracts and controls for $15 \mathrm{~min}$, the epidermis samples were washed with sterile PBS and then incu-

bated in the maintenance medium. After $42 \mathrm{~h}$, the medium was collected and frozen at $-20^{\circ} \mathrm{C}$ for further determination of IL-1a. Cell viability was determined by the MTT assay. Viability was cal- culated, considering $100 \%$ for the negative control. The histology of models was also evaluated as described below.

\subsubsection{Ocular irritation test}

The SkinEthic ${ }^{\text {TM }}$ HCE model was used and the assay performed according to the manufacturer instructions and protocol. Nega- tive (PBS-treated) and positive controls (ethanol) were used. After exposing to the extracts and controls for $10 \mathrm{~min}$, the epidermis samples were washed with sterile PBS and then incubated in the 
maintenance medium for $16 \mathrm{~h}$. After this time, the medium was collected and frozen at $-20^{\circ} \mathrm{C}$ for further determination of IL- $1 a$, and the cell viability was determined by the MTT assay. Viability was

calculated, considering $100 \%$ for the negative control. The model was also analysed regarding the histological aspects.

\subsubsection{MTT assay}

Immediately after rinsing, the tissues were evaluated for cell via- bility using the MTT assay, where yellow MTT is reduced to purple formazan primarily by enzymes (reductases) located in the mito- chondria of living cells. A stock solution of MTT was prepared in

maintenance media (provided with tissues) just prior to use and warmed to $37{ }^{\circ} \mathrm{C}$ in a water bath. Tissues were transferred to $24-$

well plates (Corning Inc., Corning, New York) containing $300 \mu \mathrm{L}$ MTT medium per well and placed in the $37^{\circ} \mathrm{C}, 5 \% \mathrm{CO}_{2}$ humidified incubator. After $3 \mathrm{~h}$ incubation, the tissues were removed from the MTT medium and any residual MTT media on the exterior of the tis- sue insert was blotted with absorbent pads. The formazan salt was extracted from the tissues by transferring them to 24-well plates containing isopropanol. The submerged tissues were incubated $3 \mathrm{~h}$ at room temperature protected from light. The plates were shaken for approximately $15 \mathrm{~min}$ once the extraction was complete. The optical density of the extracted formazan was determined by trans- ferring $200 \mu \mathrm{L}$ of each extraction solution into a clean 96-well plate. Isopropanol was used as a blank. The plates were read using a spectrophotometer (Synergy HT Microplate Reader; BioTek Instru- ments, Inc., Winooski, VT, USA) at 570 $\mathrm{nm}$. Relative cell viability was calculated for each tissue as percent of the mean of the negative control tissues.

For both models, if the percentage of viability was $>50 \%$, the substance was predicted as Non-Irritant (EU classification: no label; GHS classification: No category); if the percentage of viability was

$\leq 50 \%$, the substance was predicted as Irritant (EU classification: R38, R41 or R36, depending on the model; GSH classification: Cat- egory 1 or Category 2) (Cotovio et al., 2010).

\subsubsection{IL-1, assay}

For assessing the release of IL- $1 a$, the culture media underneath the tissues were collected at the end of the post-incubation period and kept at $-20^{\circ} \mathrm{C}$ until measurement. The quantification of IL- $1 a$ was performed using the commercial enzyme-linked immunosorbent assay (ELISA) kit Quantikine DLA50 (R\&D Systems, UK) according to the manufacturer's instructions. Mean concentrations $(\mathrm{pg} / \mathrm{mL})$ were obtained using duplicate measurements per tissue. IL-1a released was expressed as absolute data.

\subsubsection{Histology}

After incubating in the presence of CS extracts, EpiSkin ${ }^{\mathrm{TM}}$ and SkinEthic ${ }^{\mathrm{TM}}$ HCE samples (three samples per extract) were fixed with $2 \%$ paraformaldehyde during $1 \mathrm{~h}$ and embedded in Richard-Allan Scientific $^{\mathrm{TM}}$ Neg-50 ${ }^{\mathrm{TM}}$ Frozen Section Medium (ThermoScientific ${ }^{\mathrm{TM}}$ ) before cryosections $(7 \mu \mathrm{m})$ preparation (Microm HM550 cryostat; ThermoScientific $^{\mathrm{TM}}$ ). Samples were stained with haematoxylin-eosinsafran (H\&S), and epidermal thickness was measured using image analysis with Axiovision software (Zeiss, Sartrouville, France). Each image was studied for changes to the epidermis and especially to cell morphology.

\subsection{Analysis of caffeine, HMF and CGA content in EpiSkin ${ }^{\mathrm{TM}}$}

The HPLCmethod used in thisstudy to quantify HMF, CQA and caffeine in CS extracts was based on the work of Lemos et al. (2010) and Rivelli et al. (2007). The chromatographic analysis was per- formed in a HPLC integrated system (Jasco, Japan) equipped with a PU-980 pump and a Jasco AS-950 automatic sampler with a $20 \mu \mathrm{L}$ loop. Detection was performed with a Jasco model MD-2010 multiwavelength diode-array detector (DAD). The column employed was a Luna $5 \mathrm{U}$ C18 $(5 \mu \mathrm{m}, 150 \mathrm{~mm} \times 4.60 \mathrm{~mm})$ chromatographic column (Teknokroma, Spain). The mobile phase adopted was aque- ous metaphosphoric acid solution:acetonitrile $(82: 18, \mathrm{v} / \mathrm{v})$ with a flow rate of $1 \mathrm{~mL} / \mathrm{min}$ and a column temperature of $23{ }^{\circ} \mathrm{C}$. Analytes were monitored at $275 \mathrm{~nm}, 285 \mathrm{~nm}$ and $330 \mathrm{~nm}$, respectively, for caffeine, HMF and CGA, and quantification was performed on the basis of the internal standard method. Chromatographic data were analysed using the ChromNAV Software (Jasco, Japan). Calibra- tion curves were prepared for HMF $(0.36-93.0 \mathrm{mg} / \mathrm{mL})$, chlorogenic acid $(5.0-160.0$ $\mathrm{mg} / \mathrm{mL}$ ) and caffeine $(4.25-136.0 \mathrm{mg} / \mathrm{mL})$. Analyses were carried out in duplicate. Confirmation of compounds identi- ties was performed by comparing retention times and co-elution with authentic standards and also by UV absorption spectra.

\subsection{In vivo skin irritation}

\subsubsection{Patch test}

A single blinded study was done in order to evaluate the in vivo skin irritation. Twenty healthy individuals ( 15 women and 5 men) with a mean age of $30 \pm 5$ years, without known dermatological dis- eases or allergy to substances in topical products, participated in a blind study, in accordance with Declaration of Helsinki. Informed consent was obtained from all volunteers. The volunteers were asked to not apply any topical products in the forearms $24 \mathrm{~h}$ before the beginning and throughout the test period. Additionally, solar exposure was forbidden. Along with extracts, SLS (2\%, w/v, aque- ous solution) and purified water were also assayed, respectively, as positive and negative control. Five sites were marked in the inner forearms of volunteers. Before application of patch tests, the areas on the ventral part of each forearm were marked using a skin marker, and basal values were obtained by non-invasive measuring method. After basal measures were taken, the patches were applied. Fifty microlitres of the test solutions were applied on a filter paper disc $(12 \mathrm{~mm}$, Filter Paper Discs, SmartPractice,

Phoenix, USA) and occlusion was achieved with aluminium cham- bers (12 mm, Finn Chambers, SmartPractice, Phoenix, USA). Patches were removed after $48 \mathrm{~h}$, and following a period of $2 \mathrm{~h}$ of observa- tion patch test areas were evaluated by non-invasive measuring methods and visual scoring.

\subsubsection{Visual and instrumental assessments}

The visual assessment of the degree of irritation was graded according to the following scale, previously used by Agner et al. (2000): 0 - no reaction; 1 - weak, spotty erythema; 2 - well perceptible erythema covering the total exposure area; 3 - moderate erythema or severe erythema that covers the total exposure area. The same scale was used for oedema: 0 - no reaction; 1 - weak, spotty oedema; 2 - well perceptible oedema covering the total exposure area; 3 -moderate or severe oedema that covers the total exposure area (Agner et al., 2000).

Non-invasive biophysical measurements were also performed. All measurements were made with controlled temperature $\left(21-22{ }^{\circ} \mathrm{C}\right)$ and relative humidity (45-55\%). Transepidermal water

loss (TEWL) is traditionally used to assess skin barrier function (Wa and Maibach, 2010). Barrier function was evaluated by TEWL measurements, carried out with a Tewameter ${ }^{\circ}$ TM 210 (Courage Khazaka, Electronic $\mathrm{GmbH}$, Cologne, Germany), which measures the relative humidity percentage which diffuses from the dermis to the skin surface $\left(\mathrm{g} / \mathrm{cm}^{2} \mathrm{~h}\right)$.

\subsection{Statistical analysis}

Data were reported as mean \pm standard deviation of at least trip- licate experiments. Statistical analysis of the results was performed 
Table 1

EpiSkin $^{\mathrm{TM}}$ tissues were exposed for $15 \mathrm{~min}$ to controls and extracts. Cell viability was assessed by MTT while the post-exposure basal media was analysed for IL-1a release $(\mathrm{pg} / \mathrm{mL})$.

\begin{tabular}{lcc}
\hline Extract & Viability $(\%)$ & IL-1 $a(\mathrm{pg} / \mathrm{mL})$ \\
\hline W & $117.7 \pm 11.7 \mathrm{a}$ & $8.5 \pm 0.1 \mathrm{a}$ \\
WA & $105.9 \pm 15.9 \mathrm{a}$ & $76.4 \pm 2.0 \mathrm{~b}$ \\
A & $117.8 \pm 9.8 \mathrm{a}$ & $17.6 \pm 3.2 \mathrm{a}$ \\
PC (SLS) & $11.6 \pm 2.4 \mathrm{~b}$ & $522.9 \pm 32.1 \mathrm{c}$ \\
NC(PBS) & $100 \pm 9.7 \mathrm{a}$ & $28.4 \pm 2.6 \mathrm{a}, \mathrm{b}$ \\
\hline
\end{tabular}

Water (W), hydro-alcoholic (WA) mixture, alcohol (A), a positive control (PC: SLS - sodium lauryl sulfate) and a negative control (NC: PBS - phosphate buffered saline) were used. Values are expressed as mean \pm SD $(n=3)$. Values in the same column followed by different letters indicate significant differences $(p<0.05)$.

with the software SPSS 22.0 (SPSS Inc., Chicago, IL, USA). One-way ANOVA was used to investigate the differences between differ-ent extracts for all assays. Post hoc comparisons were performed according to Tukey's HSD test. In all cases, $p<0.05$ was accepted as denoting significance.

\section{Results anddiscussion}

\subsection{Skin irritability tests}

Irritation is defined as the "production of reversible damage to the skin following the application of a test substance for up to $4 \mathrm{~h}$," therefore, the more significant the initial injury (cell death), the stronger the irritant effect (UNECE, 2011). In order to confirm the potential of the extracts for topical use, irritability assays with EpiSkin $^{T M}$ test were performed. EpiSkin ${ }^{T M}$ is an alternative in vitro method efficient in predicting epidermal alterations in vivo caused by irritants. Data for the cell viability and the IL-1a release from in vitro assays with reconstituted human epidermis are shown in Table 1.

The method distinguishes between irritants and non-irritants. Irritant chemicals are identified by their ability to decrease cell via- bility below defined threshold levels of $50 \%$. The three extracts are not skin irritants for topical use in cosmetic formulations as the via- bility in all cases is higher than $50 \%$, with values ranging between

$105.9 \pm 15.9$ and $117.8 \pm 9$. 8 . This potential use was also confirmed by the low release of IL-1 $a$ as endpoint of the irritation process (in the range of negative control), which is a highly active and proinflammatory cytokine playing a key role in inflammation, being produced by keratinocytes. IL-1 $a$ is a highly active and pleiotropic proinflammatory cytokine that play a key role in inflammation, being the biological mirror of skin irritation (Balboa et al., 2014). Keratinocytes producesIL-1 $a$ and IL-1 mRNAinvitro, butonlyIL- $1 a$ biological activity has been identified in keratinocytes cultures (Mizutani et al., 1991). According to Zhang et al. (2011), the con- centration of IL1- $a$ released by keratinocytes in cultured medium increased significantly follower the exposure to different irritants. The IL-1a content, expressed as $\mathrm{pg} / \mathrm{mL}$, was $28.4 \pm 2.6$ for PBS, $522.9 \pm 32.1$ for SLS, and $8.5 \pm 0.1,76.4 \pm 2.0$ and $17.6 \pm 3.2$, respectively, for aqueous, hydro-alcoholic and alcoholic extract of CS. The absence of skin-irritant effects in extracts tested indicated that CS extracts could be safe for topical use.

The morphology alteration that occurs after the contact between the extracts and the model was evaluated for all samples and con- trols. Fig. 1 shows the effects of hydro-alcoholic extract and controls on the morphology of the treated tissues. No morphological dif- ferences were observed for the treated tissues with extracts as compared to the negative control (PBS). In contrast, the adverse effects observed with positive control in all epidermal layers con- firm the viability measurements observed. Results suggest that
Table 2

Quantification of HMF, CGA and CAF in aqueous (W), hydro-alcoholic (WA) and alcoholic (A) extracts of CS medium after EpiSkin ${ }^{\mathrm{TM}}$ assay.

\begin{tabular}{llll}
\hline Extract & HMF $(\mu \mathrm{g} / \mathrm{mL}$ extract $)$ & $\begin{array}{l}\text { CGA }(\mu \mathrm{g} / \mathrm{mL} \\
\text { extract })\end{array}$ & $\begin{array}{l}\text { Caffeine }(\mu \mathrm{g} / \mathrm{mL} \\
\text { extract })\end{array}$ \\
\hline W & $2.28 \pm 0.18$ & - & $1.26 \pm 0.06$ \\
WA & $2.39 \pm 0.27$ & - & $1.54 \pm 0.14^{*}$ \\
A & $2.26 \pm 0.09$ & - & $1.40 \pm 0.07$ \\
\hline
\end{tabular}

Values are expressed as mean $\pm \quad \mathrm{SD}(n=3)$.

Significant difference $(p<0.05)$.

histological analysis did not assess alterations to the epidermis in human skin samples after exposure to CS extracts.

There are very few reports of irritation potential in skin mod- els using extracts as this model was recently validated by ECVAM. Balboa et al. (2014) evaluated the in vitro antioxidants properties and the skin irritant effects of natural extracts obtained from under- utilized and residual vegetal and macroalgal biomass. Extracts did not affect the human reconstructed epidermis but the cell via- bility was lower than in this study. Regarding the IL-1a release, the irritation process seems to be lower. This could be justified by the different solvents used in both studies. Considering the extract composition, reported by Rodrigues et al. (2015), it is also expected no effect on skin cells, since the identified compounds did not inter- act with cells, presenting an high antioxidant activity and absence of cytotoxicity in keratinocytes and fibroblast. Also, washing proce- dures could not remove all materials and even lead to mechanical damage to the tissues, which results in an impaired prediction of the true skin irritation potential of the materials (Molinari et al., 2013).

\subsection{Analytical methods}

A further part of the study was concerned with the analysis of caffeine, CGA and HMF in the medium after the skin in vitro model assay for the three extracts. Chro- matograms of CS extracts (A) and standards (B) are depicted in Fig. 2. Compounds were numbered according to their elution order.

HPLC analysis of the CS extracts indicated the presence of caffeine and HMF and the absence of CGA for all extracts. The quan- tification of CAF and $\mathrm{HMF}$ is reported in Table 2 .

Caffeine content in CS extracts ranged between $1.26 \pm 0.06 \mu \mathrm{g} / \mathrm{mL}$ and $1.54 \pm 0.14 \mu \mathrm{g} / \mathrm{mL}$ for aqueous and hydroalcoholic ones, respectively. There is a statistical difference between the caffeine content of hydro-alcoholic extract and the other extracts. As previous mentioned, determination of caffeine in CS is very important, since they have a great effect on the final quality of the extracts used for cosmetic proposes. Previously work done in our laboratory, evaluated the CGA content in CS hydro-alcoholic extract and results demonstrated the presence of this compound (Pereira, 2012). The absence observed in the present study is justified by the retention effect of the epidermal model, which prevents the compounds pas- sage. To the best of our knowledge, this is the first study that reports the content of caffeine after an EpiSkin ${ }^{T M}$ assay using extracts.

\subsection{Ocular irritability tests}

The assessment of ocular irritation is part of the early testing procedure for the evaluation of new cosmetic ingredients (Cotovio et al., 2010). The use of cosmetic products can produce adverse effects on the ocular surface, ranging from mild discomfort to visionthreatening conditions (Coroneo et al., 2006). These compli- cations can be related to allergy or toxicity (Coroneo et al., 2006). 


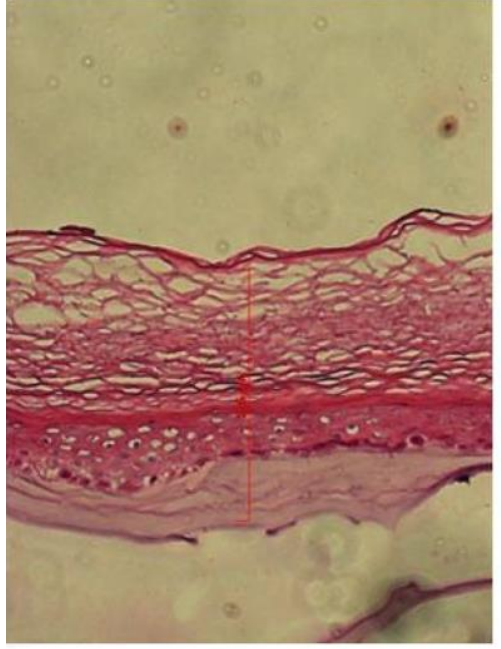

a

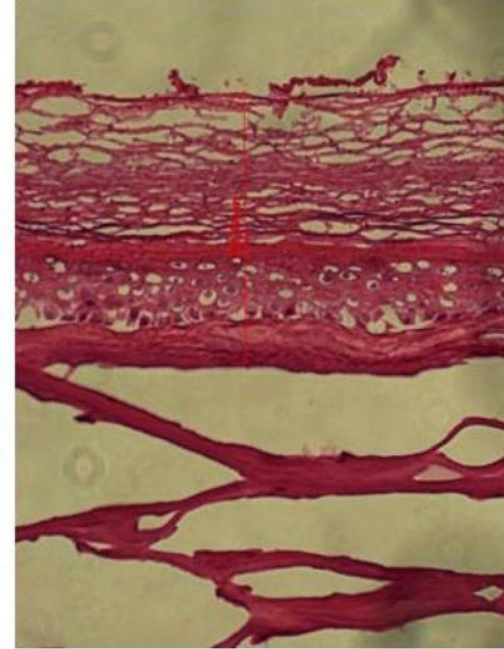

b

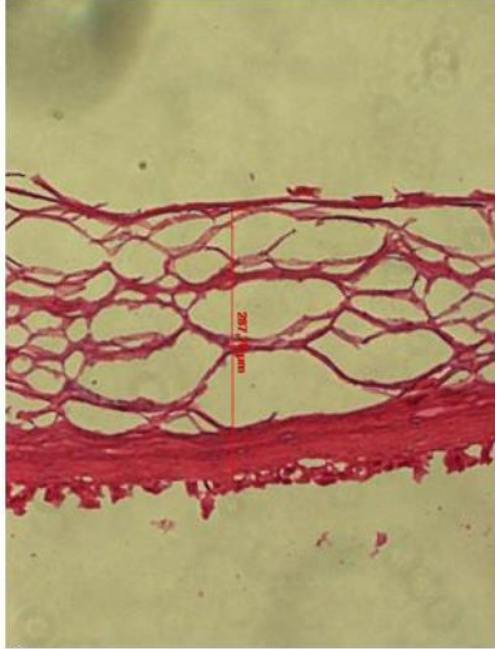

C

Fig. 1. Haematoxylin-eosin (HE) staining of the EpiSkin ${ }^{\mathrm{TM}}$ tissues. (a) After topical application of $10 \mu \mathrm{L}$ of hydro-alcoholic extract of CS: no irritation signs. (b) Negative control (100\% cell viability): no irritation signs. (c) Positive control ( $5 \%$ SDS): marked epidermolysis.

In this field, numerous non-animal test systems have been devel- oped over the years (OECD, 2009a,b). SkinEthic ${ }^{T M}$ HCE is one of the two reconstructed human tissue that are currently available for the purpose of eyeirritationandisundervalidation byECVAM(Alépée et al., 2013).

In order to evaluate the eye irritation potential of CS extracts, a SkinEthic ${ }^{T M}$ HCE test was done. Table 3 summarizes the results for cell viability and release of IL-1a observed with extracts and controls.

a
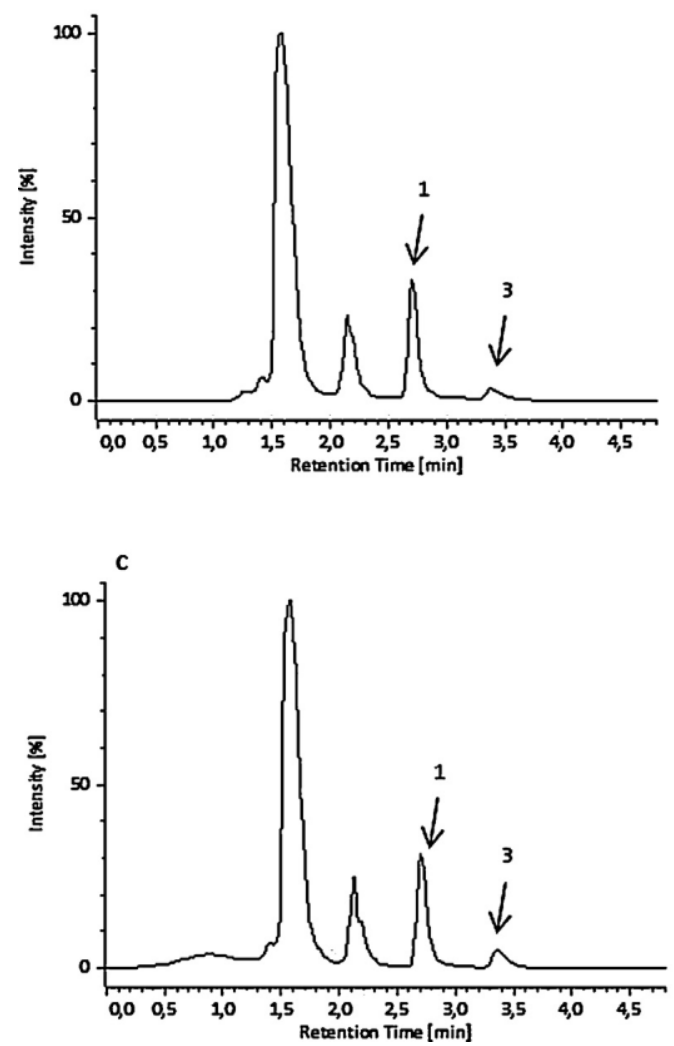

The viability of cells with aqueous and hydro-alcoholic extracts were higher than $50 \%$, with percentages of $132.6 \pm 21.3$ and

$123.6 \pm 13.7$, respectively, which means that the extracts were classified as non-irritants for the eyes. Ethanolic extract presented a percentage of viability of $48.0 \pm 7.5$, being in the borderline between irritant and non irritant, which is justify by the higher amount of alcohol used. Regarding the IL-1a release, no significant differences were observed between negative control and aqueous or hydro-alcoholic extracts. However, the release of IL-1 $a$ slightly

b

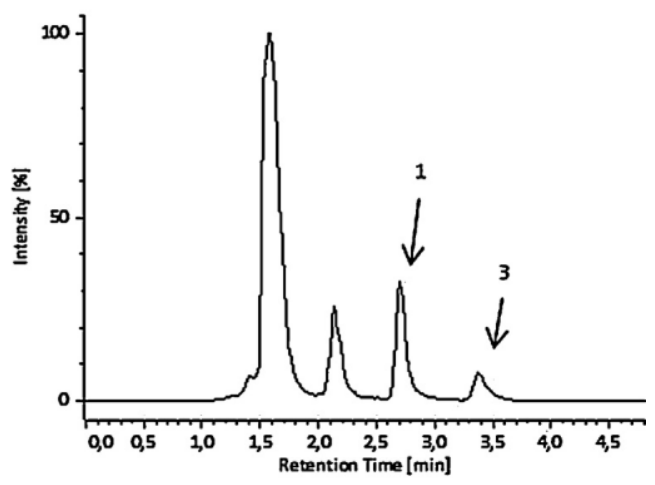

1

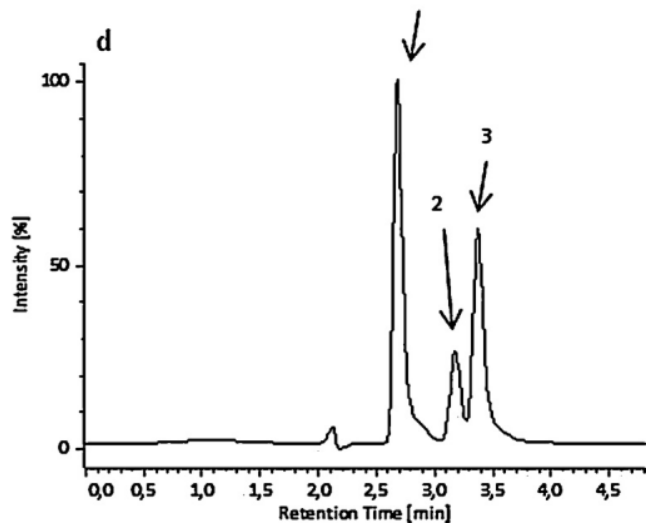

Fig. 2. Chromatograms of aqueous extract (a), hydro-alcoholic extract (b) and alcoholic extract (c) of CS and a standard mixture (1 mg/mL) (d) of HMF (1), CQA (2) and caffeine (3). 

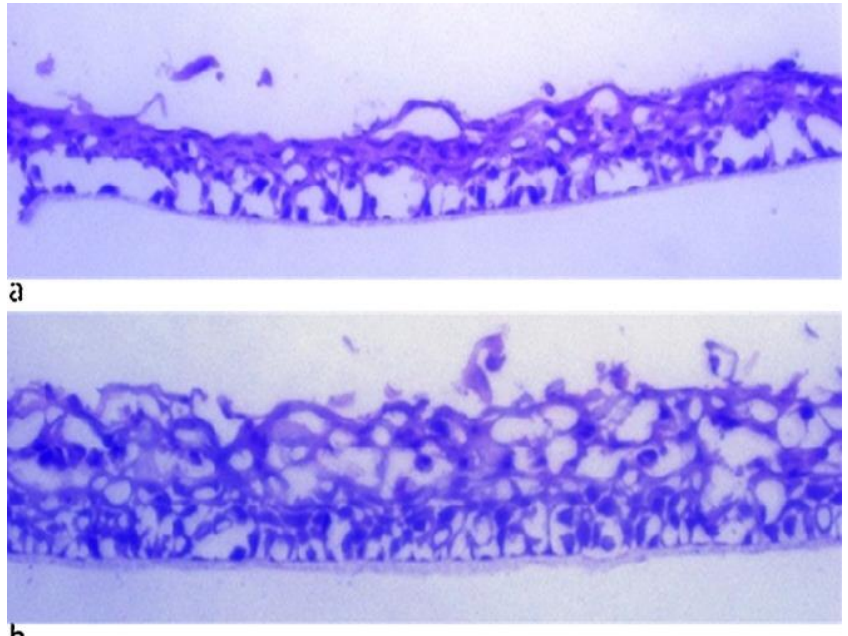

b

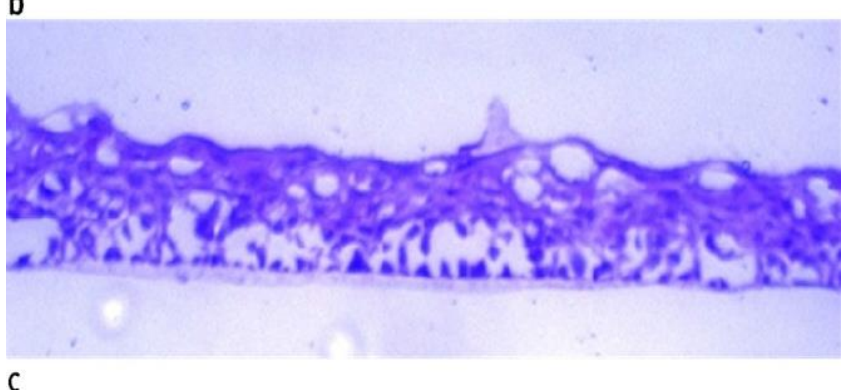

Fig. 3. Haematoxylin-eosin (HE) staining of the SkinEthic ${ }^{\text {TM }}$ HCE tissues. After top- ical application of $10 \mu \mathrm{L}$ of alcoholic (a) extract of CS: irritation signals. (b) Negative control ( $100 \%$ cell viability): no irritation signs. (c) Positive control ( $5 \%$ SLS): marked epidermolysis.

increased when using the positive control and ethanol extract as compared to the negative control. As mentioned by manufacture, ethanol was used as negative control.

The histological images obtained after topical application of the aqueous and hydro-alcoholic extracts of CS also confirms that both are not irritant, being good candidates for cosmetic ingredients. Regarding alcoholic extract, results are very different. As it is pos- sible to confirm, this extract leads to histological alterations in SkinEthic ${ }^{\mathrm{TM}}$ HCE tissues, similarly to positive control (Fig. 3).

As far as we know, this is also the first time that SkinEthics ${ }^{\mathrm{TM}}$ HCE is used to evaluate the ocular irritation for food by-products extracts in order to incorporate them in cosmetics as active ingredients.

\subsection{In vivo test}

According to Miles et al. (2014), reconstructed human epider- mis like EpiSkin ${ }^{\mathrm{TM}}$ has some limitations. This model is composed

\section{Table 3}

SkinEthic ${ }^{\text {TM }}$ HCE model was exposed for 10 min to controls and extracts. Cell viability was assessed by MTT while the post-exposure basal media was analysed for IL-1a release $(\mathrm{pg} / \mathrm{mL})$.

\begin{tabular}{lcc}
\hline Extract & Viability $(\%)$ & $\mathrm{IL}-1 a(\mathrm{pg} / \mathrm{mL})$ \\
\hline W & $132.6 \pm 21.3 \mathrm{a}$ & $41.4 \pm 2.5 \mathrm{a}$ \\
WA & $123.6 \pm 13.7 \mathrm{a}$ & $34.2 \pm 1.6 \mathrm{a}$ \\
A & $48.0 \pm 7.5 \mathrm{~b}$ & $82.2 \pm 22.9 \mathrm{~b}$ \\
PC (ethanol) & $2.9 \pm 0.5 \mathrm{c}$ & $114.4 \pm 2.5 \mathrm{c}$ \\
NC(PBS) & $86.0 \pm 6.1 \mathrm{~d}$ & $23.7 \pm 1.0 \mathrm{a}$ \\
\hline
\end{tabular}

Water (W), hydro-alcoholic (WA) mixture, alcohol (A), a positive control (PC) and a negative control (NC: PBS - phosphate buffered saline) were used. Values are expressed as mean \pm SD $(n=3)$. Values in the same column followed by different letters indicate significant differences $(p<0.05)$.

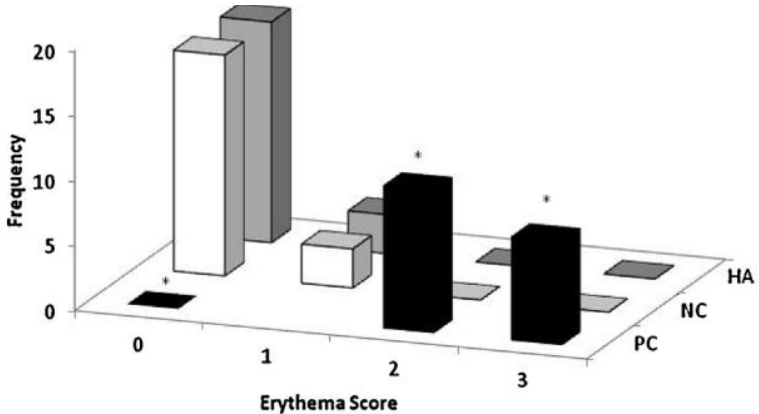

A

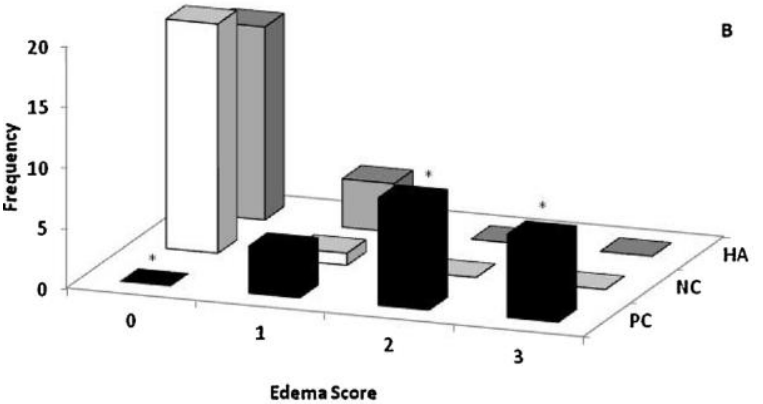

- $P C$

$\square N C$

$\square \mathrm{HA}$

Fig. 4. Frequency of clinical scores attributed to positive control (PC - SLS), nega- tive control (NC - purified water) and hydro-alcoholic extract of CS (HA) regarding erythema and oedema after patch removal. ${ }^{*}$ means a significant difference $(p<0.05)$.

of a highly differentiated multi-layer human epidermis cells, but are totally lacking in any network of the pilo-sebaceous units, which regulate dermal homeostasis (Miles et al., 2014). Also, the hydrolipid film cannot be totally reproduced regarding its bio- physiological properties of components (urea, glycolic acid) neither to the occlusive effect of the film's lipid component (ceramides) (Miles et al., 2014). Some cells normally present in human skin are not present in this model such as dendritic Langerhans' cells. The higher penetration rate of these tissues makes these skin models more sensitive to test compounds (Perkins et al., 1999), which could result in over-prediction of strong irritants (Welss et al., 2004). Thus, to ensure that the extract is completely safe, some other assays like patch test should be done. Patch testing after a single application is a widely used procedure to evaluate acute irritant reactions in human volunteers.

The previous assays led us to select the hydro-alcoholic extract as the best one to be incorporated in cosmetic products, as it did not affect the morphology of skin and ocular models, with high cell viability for both assays and low IL- $1 a$ release. Also the CAF con- tent was higher than in the other extracts evaluated and, according to Rodrigues et al. (2015), the antioxidant content and antimi- crobial activity is higher. Considering not only these results but also sustainability questions and other previous works regarding the antioxidant activity of these extracts, hydro-alcoholic extract seems to be the best one, being selected for the final patch test (Bresciani et al., 2014; Costa et al., 2014; Narita and Inouye, 2012).

\subsubsection{Skin compatibility by evaluation of primary skin irritation}

Initially, evaluation of irritancy testing was based on visual score. Results obtained by visual analyse are shown in Fig. 4. Results of patch testing demonstrated that hydro-alcoholic provoked a slight erythema in three volunteers $2 \mathrm{~h}$ after the patch test removal, however, when statistical analyses were applied regarding negative control, no differences were found. Regarding the positive control, it is possible to observe statistical differences when comparing with negative control and hydro-alcoholic extract $(p<0.05)$. 


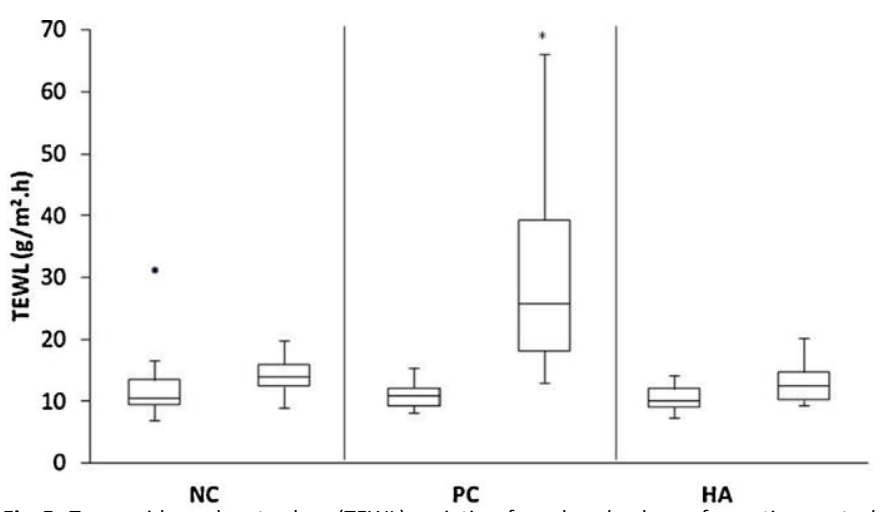

Fig. 5. Transepidermal water loss (TEWL) variation from basal values of negative control (NC), positive control (PC) and hydro-alcoholic extract (HA). The left boxes represent values at time 0 and the right boxes represent values $2 \mathrm{~h}$ after patch removal. Outliers are presented as circles. ${ }^{*}$ means a significant difference $(p<0.05)$.

\subsubsection{Transepidermal water loss variation}

The measurement of transepidermal water loss (TEWL) is a wellestablished method in dermatology to assess the integrity of the skin barrier in vivo and an important measure of epidermal barrier function (Pinnagoda et al., 1990). When skin is damaged, the barrier function is impaired which results in a higherwaterloss. The extract did not alter the skin barrier function, as TEWL values were not changed, when compared to the positive control (Fig. 5).

No significant differences were observed for TEWL measurements, in comparison with purified water, $2 \mathrm{~h}$ after patch removal $(p<$ $0.05)$, which indicates the absence of barrier disruption. Consequently, CS hydro-alcoholic extract is not potentially irritating. The only effect on TEWL values was probably due to the even- tual formation of a greasy film by the lipophilic components of the extract. Comparing with other extracts that are under investiga- tion, CS could be consider as safe for cosmetic purposes (Almeida et al., 2008; Dal'Belo et al., 2006). Unlike arnica and Calendula offic- inalis, CS did not originate skin irritation (Reider et al., 2001). As expected, SLS (PC) leads to an increase of TEWL values.

\section{Conclusion}

This study constitutes an objective evaluation of the safety of CS extract as cosmetic ingredient and contributes to the elucidation of its mechanism of action. It is an overview of the effect of CS extracts in a validated human skin model for irritancy and eye model under validation. In vitro studies on ocular and dermal irritation were car- ried out with three different extracts of CS. The in vivo study was carried out with the extract that revealed the best results for the in vitro assays. Results of in vitro studies revealed that CS extracts are safe regarding to skin and ocular irritancy as cell viability was equal to the negative control in both assays and the IL-1a was under $50 \%$. The histological analysis demonstrated that extracts did not affect the skin neither the ocular model. Quantitative chromato- graphic investigations revealed that the three extracts contained caffeine and $\mathrm{HMF}$, but there were no traces of CGA. The in vivo patch tests proved that the hydro-alcoholic extract did not cause skin irri- tation. The next steps to evaluate the safety of the extract would undergo sensitization and tolerance studies in normal condition of use.

\section{Acknowledgements}

The authors greatly acknowledge all volunteers. Francisca Rodrigues and Rita Alves are thankful to Foundation for Science and Technology (Portugal) for a Ph.D. grant (SFRH/BDE/51385/2011) and a Post-doc grant (SFRH/BPD/68883/2010), respectively, financed by POPH-QREN and subsidized by European Science Foun- dation.

This work received financial support from the European Union (FEDER funds through COMPETE) and National Funds (FCT, Foundation for Science and Technology) through project PestC/EQB/LA0006/2013. The work also received financial support from the European Union (FEDER funds) under the framework of QREN through Project NORTE-07-0124-FEDER-000069. To all financing sources the authors are greatly indebted.

\section{References}

Agner, T., Held, E., West, W., Gray, J., 2000. Evaluation of an experimental patch test model for the detection of irritant skin reactions to moisturisers. Skin Res. Technol. 6, 250-254.

Alépée, N., Bessou-Touya, S., Cotovio, J., de Smedt, A., de Wever, B., Faller, C., Jones, P., Le Varlet, B., Marrec-Fairley, M., Pfannenbecker, U., Tailhardat, M., van Goethem, F., McNamee, P., 2013. Cosmetics Europe multi-laboratory pre-validation of the SkinEthic ${ }^{\mathrm{TM}}$ reconstituted human corneal epithelium test method for the prediction of eye irritation. Toxicol. In Vitro 27, 1476-1488.

Almeida, I.F., Valentão, P., Andrade, P.B., Seabra, R.M., Pereira, T.M., Amaral, M.H., Costa, P.C., Bahia, M.F., 2008. In vivo skin irritation potential of a Castanea sativa (Chestnut) leaf extract, a putative natural antioxidant for topical application. Basic Clin. Pharmacol. Toxicol. 103, 461-467.

Alves, R.C., Casal, S., Oliveira, M.B.P.P., 2009. Tocopherols in espresso coffee: analyt- ical method development and validation. Food Chem. 115, 1549-1555.

Balboa, E.M., Soto, M.L., Nogueira, D.R., González-López, N., Conde, E., Moure, A., Vinardell, M.P., Mitjans, M., Domínguez, H., 2014. Potential of antioxidant extracts produced by aqueous processing of renewable resources for the for- mulation of cosmetics. Ind. Crops Prod. 58, 104-110.

Bolzinger, M.A., Briancon, S., Pelletier, J., Fessi, H., Chevalier, Y., 2008. Percutaneous release of caffeine from microemulsion, emulsion and gel dosage forms. Eur. J. Pharm. Biopharm. 68, 446-451.

Borrelli, R.C., Esposito, F., Napolitano, A., Ritieni, A., Fogliano, V., 2004. Characteri- zation of a new potential functional ingredient: coffee silverskin. J. Agric. Food Chem. 52 , 1338-1343.

Bresciani, L., Calani, L., Bruni, R., Brighenti, F., Del Rio, D., 2014. Phenolic composition, caffeine content and antioxidant capacity of coffee silverskin. Food Res. Int. 61, 196-201.

Capuano, E., Fogliano, V., 2011. Acrylamide and 5-hydroxymethylfurfural (HMF): a review on metabolism, toxicity, occurrence in food and mitigation strategies. LWT Food Sci. Technol. 44, 793-810.

Coroneo, M.T., Rosenberg, M.L., Cheung, L.M., 2006. Ocular effects of cosmetic prod- ucts and procedures. Ocul. Surf. 4, 94-102.

Costa, A.S.G., Alves, R.C., Vinha, A.F., Barreira, S.V.P., Nunes, M.A., Cunha, L.M., Oliveira, M.B.P.P., 2014. Optimization of antioxidants extraction from coffee sil- verskin, a roasting by-product, having in view a sustainable process. Ind. Crops Prod. 53, 350357.

Cotovio, J., Grandidier, M.H., Lelièvre, D., Bremond, C., Amsellem, C., Maloug, S., Ovi- gne, J.M., Loisel-Joubert, S., Lee, A.V.D., Minondo, A.M., Capallere, C., Bertino, B., Alépée, N., Tinois-Tessonneaud, E., de Fraissinette, A.D.B., Meunier, J.R., Leclaire, J., 2010. In vitro assessment of eye irritancy using the reconstructed human corneal epithelial SkinEthic ${ }^{\text {TM }}$ HCE model: application to 435 substances from consumer products industry. Toxicol. In Vitro 24, 523-537.

Dal'Belo, S.E., Rigo Gaspar, L., Berardo Gonçalves Maia Campos, P.M., 2006. Mois- turizing effect of cosmetic formulations containing Aloe vera extract in different concentrations assessed by skin bioengineering techniques. Skin Res. Technol. 12, 241-246.

del Campo, G., Berregi, I., Caracena, R., Zuriarrain, J., 2010. Quantitative determi- nation of caffeine, formic acid, trigonelline and 5-(hydroxymethyl)furfural in soluble coffees by ${ }^{1} \mathrm{H}$ NMR spectrometry. Talanta $81,367-371$.

Draize, J.H., Woodward, G., Clavery, H.O., 1944. Methods for the study of irritation and toxicity of substances applied topically to the skin and mucous membranes. J. Pharmacol. Exp. Ther. 82, 377-390.

Herman, A., Herman, A.P., 2013. Caffeine's mechanisms of action and its cosmetic use. Skin Pharmacol. Physiol. 26, 8-14.

Lemos, G., dos Santos, J.S., dos Santos, M.L.P., 2010. Method validation to HMF determination in honey by HPLC-UV and its influence on the product quality. Quim. Nova 33, 1682-1685.

Ludwig, I.A., Sanchez, L, Caemmerer, B., Kroh, L.W. De Peñ a, M.P., Cid, C., 2012. Extraction of coffee antioxidants: impact of brewing time and method. Food Res. Int. $48,57-64$.

Miles, A., Berthet, A., Hopf, N.B., Gilliet, M., Raffoul, W., Vernez, D., Spring, P., 2014. A new alternative method for testing skin irritation using a human skin model: a pilot study. Toxicol. In Vitro 28, 240-247.

Mills, C.E., Oruna-Concha, M.J., Mottram, D.S., Gibson, G.R., Spencer, J.P.E., 2013. The effect of processing on chlorogenic acid content of commercially available coffee. Food Chem $141,3335-3340$.

Mizutani, H., Black, R., Kupper, T.S., 1991. Human keratinocytes produce but do not process pro-interleukin-1 (IL-1) beta. Different strategies of IL-1 production and processing in monocytes and keratinocytes. J. Clin. Invest. 87, 1066-1071. 
Molinari, J., Eskes, C., Andres, E., Remoué, N., Sá-Rocha, V.M., Hurtado, S.P., Bar- richello, C., 2013. Improved procedures for in vitro skin irritation testing of sticky and greasy natural botanicals. Toxicol. In Vitro 27, 441-450.

Narita, Y., Inouye, K., 2012. High antioxidant activity of coffee silverskin extracts obtained by the treatment of coffee silverskin with subcritical water. Food Chem. 135, 943-949.

OECD, 2009a. Guidelines for the Testing of Chemicals No. 437. The Bovine Corneal Opacity and Permeability (BCOP) Test Method for Identifying Ocular Corrosives and Severe Irritants. Organisation for Economic Cooperation and Development, Paris, France, pp. 18.

OECD, 2009b. Guidelines for the Testing of Chemicals No. 438. The Isolated Chicken Eye

(ICE) Test Method for Identifying Ocular Corrosives and Severe Irritants. Organisation for Economic Cooperation and Development, Paris, France, pp. 18. Pandey, A., Soccol, C.R.,

Nigam, P., Brand, D., Mohan, R., Roussos, S., 2000. Biotechno- logical potential of coffee pulp

J. 6, 153-162.

and coffee husk for bioprocesses. Biochem. Eng.

Panusa, A., Zuorro, A., Lavecchia, R., Marrosu, G., Petrucci, R., 2013. Recovery of natu- ral antioxidants from spent coffee grounds. J. Agric. Food Chem. 61, 4162-4168. Pereira, S., (Master Thesis) 2012. Caracterização da pele de prata no contexto da valorização de versity of Porto, Porto, pp. 84 produtos da indústria agroalimentar. Quality Control, Uni-

Perkins, M.A., Osborne, R., Rana, F.R., Ghassemi, A., Robinson, M.K., 1999. Compar- ison of in vitro and in vivo human skin responses to consumer products and ingredients with a range of irritancy potential. Toxicol. Sci. 48, 218-229.

Pinnagoda, J., Tupkek, R.A., Agner, T., Serup, J., 1990. Guidelines for transepidermal water loss (TEWL) measurement. Contact Dermatitis 22, 164-178.

Pourfarzad, A., Mahdavian-Mehr, H., Sedaghat, N., 2013. Coffee silverskin as a source of dietary fiber in bread-making: optimization of chemical treatment using response surface methodology. LWT Food Sci. Technol. 50, 599-606.

Reider, N., Komericki, P., Hausen, B.M., Fritsch, P., Aberer, W., 2001. The seamy side of natural medicines: contact sensitization to arnica (Arnica montana L.) and marigold (Calendula officinalis L.). Contact Dermatitis 45, 269-272.
Rivelli, D.P., Ropke, C.D., Silva, V.V., Miranda, D.V., Almeida, R.L., Sawada, T.C.H., Barros, S.M.B., 2007. Simultaneous determination of chlorogenic acid, caffeic acid and caffeine in hydroalcoholic and aqueous extracts of llex paraguarien-sis by HPLC and correlation with antioxidant capacity of the extracts by DPPH reduction. Braz. J. Pharm. Sci. 43, 215-222.

Rodrigues, F., Palmeira-de-Oliveira, A., Das Neves, J., sarmento, B., Amaral, M.H., Oliveira, M.B.P.P., 2015. Coffee silverskin: a possible valuable cosmetic ingredi- ent. Pharm Biol., http://dx.doi.org/10.3109/13880209.2014.922589 (in press).

Saenger, M., Hartge, E.U., Werther, J., Ogada, T., Siagi, Z., 2001. Combustion of coffee husks. Renew. Energy 23, 103-121.

Sato, Y., Itagaki, S., Kurokawa, T., Ogura, J., Kobayashi, M., Hirano, T., Sugawara, M., Iseki, K., 2011. In vitro and in vivo antioxidant properties of chlorogenic acid and caffeic acid. Int. J. Pharm. 403, 136-138.

Spielmann, H., Hoffmann, S., Liebsch, M., Botham, P., Fentem, J.H., Eskes, C., Roguet, R., Cotovio, J., Cole, T., Worth, A., Heylings, J., Jones, P., Robles, C., Kandarova, H., Gamer, A., Remmele, M., Curren, R., Raabe, H., Cockshott, A., Gerner, I., Zuang, V., 2007. The ECVAM international validation study on in vitro tests for acute skin irritation: report on the validity of the EPISKIN and EpiDerm assays and on the Skin Integrity Function Test. Altern. Lab. Anim. 35, 559-601.

Tornier, C., Amsellem, C., Fraissinette, A.B., Alépée, N., 2010. Assessment of the opti- mized SkinEthic Reconstructed Human Epidermis (RHE) 42 bis skin irritation protocol ove 39 test substances. Toxicol. In Vitro 24, 245-256.

UNECE, 2011. Skin corrosion/irritation. In: Globally Harmonized System of Classifi-cation and Labelling of Chemicals (GHS), fourth ed. United Nations Department of Labor, New York, Geneva.

Wa, C.V., Maibach, H.I., 2010. Mapping the human face: biophysical properties. Skin Res. Technol. 16, 38-54.

Welss, T., Basketter, D.A., Schröder, K.R., 2004. In vitro skin irritation: facts and future. State of the art review of mechanisms and models. Toxicol. In Vitro 18, 231-243. Zhang, Q., Dai, T., Zhang, L., Zhang, M., Xiao, X., Hu, H., Zou, P., Liu, X., Xiang, Q., Su, Z., Huang, Y., He, Q.Y., 2011. Identification of potential biomarkers for predicting acute dermal irritation by proteomic analysis. J. Appl. Toxicol. 31, 762-772. 